\title{
openheart Variability of cardiovascular magnetic resonance (CMR) T1 mapping parameters in healthy volunteers during long-term follow-up
}

Fabian aus dem Siepen, ${ }^{1}$ Christian Baumgärtner, ${ }^{1}$ Matthias Müller-Henessen, ${ }^{1}$
Florian André,,${ }^{1}$ Daniel Messroghli, ${ }^{2}$ Marco Ochs, ${ }^{1}$ Johannes Riffel, ${ }^{1}$
Evangelos Giannitsis, ${ }^{1}$ Hugo A Katus, ${ }^{1}$ Matthias G Friedrich,,${ }^{1}$ Sebastian J Buss ${ }^{1}$

\section{ABSTRACT}

Background Myocardial $\mathrm{T} 1$ and extracellular volume (ECV) derived from cardiovascular MRIs are more and more widely accepted as important markers for diagnosis, risk prediction and monitoring of cardiac disease. Yet data regarding long-term stability of myocardial T1 mapping are lacking. The aim of this study was to investigate the long-term stability of native and postcontrast T1 mapping values in healthy volunteers.

Methods 18 strictly selected healthy volunteers $(52 \pm 10$ years, 12 men) were studied on a Philips Achieva 1.5

Tesla scanner. T1 relaxation times were measured before and $15 \mathrm{~min}$ after a bolus contrast injection of gadolinium diethylenetriamine penta-acetic acid (DTPA) $(0.2 \mathrm{mmol} / \mathrm{kg})$ using a single-breath-hold modified Look-Locker inversion recovery 3(3)3(3)5 sequence. ECV was calculated using native and postcontrast T1 times of myocardium and blood correcting for blood haematocrit. Exams were repeated $3.6 \pm 0.5$ years later under the same conditions and using the same scan protocols.

Results Cardiac biomarkers (high-sensitivity troponin $\mathrm{T}$ and $\mathrm{N}$ terminal pro-brain natriuretic peptide) remained unchanged, as well as left ventricular mass, and global and longitudinal function. No significant change occurred regarding native T1 times $(1017 \pm 24 \mathrm{~ms}$ vs $1015 \pm 21 \mathrm{~ms}$; $\mathrm{P}=0.6$ ), postcontrast $\mathrm{T} 1$ times ( $426 \pm 38 \mathrm{~ms}$ vs $413 \pm 20 \mathrm{~ms}$; $\mathrm{P}=0.13)$ or $\mathrm{ECV}(22 \% \pm 2 \%$ vs $23 \% \pm 2 \% ; \mathrm{P}=0.3)$. Native $\mathrm{T} 1$ time and ECV appeared to be better reproducible than postcontrast $\mathrm{T} 1$, resulting in lower coefficients of variation (ECV: $3.5 \%$, native T1: $1.3 \%$, postcontrast T1: $6.4 \%$ ) and smaller limits of agreement (ECV: $2 \% /-2 \%$, native T1: 39 $\mathrm{ms} /-35 \mathrm{~ms}$, postcontrast T1: $85 \mathrm{~ms} /-59 \mathrm{~ms}$ ).

Conclusions During long-term follow-up, native T1 and ECV values are very robust markers, whereas postcontrast $\mathrm{T} 1$ results appear less stable.

\section{INTRODUCTION \\ Background}

Cardiovascular magnetic resonance (CMR) T1 mapping represents a quantitative imaging technique for the characterisation of the myocardium and identifies changes in myocardial tissue composition. T1 mapping therefore offers the opportunity to detect

\section{Key questions}

What is already known about this subject?

- Myocardial T1 and extracellular volume (ECV) derived from cardiovascular MRIs are more and more widely accepted as important markers for diagnosis, risk prediction and monitoring of cardiac disease. Yet data regarding long-term stability of myocardial $\mathrm{T} 1$ mapping are lacking.

What does this study add?

- This study investigated the long-term stability of native and postcontrast $\mathrm{T} 1$ mapping values in healthy volunteers.

How might this impact on clinical practice?

- The demonstrated long-term stability of T1 mapping parameters in this study might help to establish this technique in clinical routine.

and to quantify pathological changes of the myocardium in various heart diseases. ${ }^{1}$ In T1 mapping, parametric maps are generated where the signal intensity of each pixel represents its longitudinal or spin-lattice relaxation time $\mathrm{T} 1$ in milliseconds, which is a time constant that describes a specific magnetic tissue property. T1 maps can be assessed before (native T1) and after (postcontrast T1) administration of a gadolinium-based extracellular contrast agent. Moreover, the extracellular volume (ECV) fraction can be estimated from a combination of native and postcontrast $\mathrm{T} 1$ values.

Recently, several studies could demonstrate characteristic changes in native myocardial $\mathrm{T} 1$, postcontrast $\mathrm{T} 1$ and $\mathrm{ECV}$ in different forms of ischaemic and non-ischaemic heart diseases, including acute ${ }^{2}$ and early states of diseases. ${ }^{3}$ For instance, changes in $\mathrm{T} 1$ times can be caused by interstitial fibrosis, ${ }^{45}$ oedema $^{6}$ or amyloid deposition. ${ }^{7}$ 
The capability of this technique to quantify the amount of diffuse cardiac fibrosis could be demonstrated by comparing T1 mapping parameters with histological parameters from myocardial biopsies. ${ }^{8-10}$ Recent studies revealed even a prognostic value for $\mathrm{T} 1$ mapping in patients with aortic stenosis. ${ }^{11}$ Furthermore, T1 mapping might be able to detect early microvascular dysfunction in adenosine stress tests. ${ }^{12}$

Before a diagnostic test is implemented in clinical routine, its technical characteristics have to be investigated, in particular with regard to normal values, comparability, reproducibility and stability. Recent studies reported normal ranges for healthy subjects and data on short-term stability for 1.5 and 3 Tesla. Additionally, Piechnik $e a^{13}$ reported only small differences in native $\mathrm{T} 1$ in a cohort of 18 healthy volunteers over a period of 3 years using the shortened modified Look-Locker inversion recovery (MOLLI) technique.

\section{Study aim}

To date, it is unknown however if ECV values of healthy individuals change over time or remain constant. We sought to investigate the long-term stability of $\mathrm{T} 1$ mapping values including ECV in a cohort of well-characterised, healthy volunteers.

\section{METHODS}

\section{Study population}

Eighteen volunteers were prospectively enrolled between 2010 and 2011. Recruitment was performed using postings and newspaper advertisement, inviting apparently healthy volunteers to participate in a scientific study and following a standard protocol. Except for thyroid hormone substitution and oral contraceptives, permanent medication was a contraindication. For follow-up, these volunteers were enrolled by written invitation in 2015. All participants gave written informed consent.

\section{Clinical examination and ECG}

All subjects underwent clinical examination and 12-lead ECG at the baseline visit and at the follow-up visit. Medical history, family history, physical examination and ECG analysis were performed by two clinically experienced physicians and supervised by senior physicians.

\section{Laboratory testing}

Extensive blood analyses were performed both at the baseline visit and at the follow-up visit, including complete blood cell count, electrolytes, cardiac biomarkers, liver and kidney function tests, and thyroid hormone levels. Additionally, test subjects underwent an oral glucose tolerance test at the baseline visit. Standard assays were used for determination of troponin T (ECLIA, Roche Diagnostics) and Nterminal pro-brain natriuretic peptide (NT-proBNP) (CLIA, Siemens). Blood samples were collected on the same day as the CMR scan was performed.

\section{Cardiovascular magnetic resonance}

All scans were performed on the same Philips 1.5 Tesla MRI scanner (Achieva) with a 32-element cardiac receiver coil and vector-ECG gating. Steady-state free-precession cine sequences were applied in contiguous shortaxis and in three long-axis views (two-chamber, threechamber, four-chamber). T1 imaging was performed using a MOLLI sequence in a mid-ventricular short-axis view during breath-hold in end-expiration ${ }^{14}$ before and 15 min after administration of gadolinium diethylenetriamine penta-acetic acid /DTPA (Magnograf, $0.2 \mathrm{mmol} /$ $\mathrm{kg}$ body weight). At baseline, an adenosine stress examination was performed to exclude significant coronary artery disease.

Contiguous short-axis stack images were used to measure cardiac volumes, left ventricular ejection fraction (LVEF) and myocardial mass in late diastole using a commercially available workstation (Philips IntelliSpace, V.7.0.1, Best, The Netherlands).

Precontrast and postcontrast T1 maps were generated and analysed using a commercial software package for cardiovascular image analysis ( $\mathrm{cvi}^{42}$, V.5.2, Circle Cardiovascular Imaging, Calgary, Canada). Endocardial and epicardial borders were defined manually, using an offset of $15 \%$ to avoid partial-volume effects in the subendocardial and subepicardial layers. Global myocardial $\mathrm{T} 1$ values were calculated as a mean of all segments with respect to the segment's area. $\mathrm{T} 1$ values for blood were gathered by manually drawing a region of interest in the left ventricular (LV) cavity.

ECV values were created according to the following formula ${ }^{15}$ :

$$
E C V=(1-H C T) \frac{\left(\frac{1}{T 1_{\text {myo post }}}-\frac{1}{T 1_{\text {myo pre }}}\right)}{\left(\frac{1}{T 1_{\text {blood }_{\text {post }}}}-\frac{1}{T 1_{\text {blood }_{\text {pre }}}}\right)}
$$

\section{Statistical analysis}

Statistical analysis was performed using MedCalc V.16.4 statistical software (MedCalc Software, Ostend, Belgium).

The Kolmogorov-Smirnov test was used to test for normal distributions. Categorical data are expressed as percentages and continuous variables as mean \pm SD. For comparison of two parametric variables, the paired t-test was used. For analysis of the reproducibility of different parameters, we used Bland-Altman plots and coefficients of variation. A $\mathrm{P}$ value of less than 0.05 was considered statistically significant.

\section{RESULTS}

\section{Clinical parameters and laboratory results}

No study subjects reported symptoms or any other evidence of cardiac disease at baseline and after follow-up (mean interval $3.8 \pm 0.6$ years). None of the subjects had medications on a regular basis. All clinical and ECG values were within the normal range at baseline as well as at the follow-up visit. There was a small yet significant increase of systolic (from $119 \pm 10$ to $126 \pm 9 \mathrm{~mm} \mathrm{Hg}$; 


\begin{tabular}{lllc}
\hline Table 1 & Clinical characteristics and ECG data & \\
\hline & $\begin{array}{l}\text { Baseline } \\
\text { visit }\end{array}$ & $\begin{array}{l}\text { Follow-up } \\
\text { visit }\end{array}$ & P value \\
\hline Height (cm) & $174 \pm 11$ & $174 \pm 11$ & 0.67 \\
Weight (kg) & $76 \pm 16$ & $76 \pm 16$ & 0.42 \\
\hline Body mass index (kg/m $\left.{ }^{2}\right)$ & $25 \pm 3$ & $25 \pm 3$ & 0.43 \\
\hline Age (years) & $54 \pm 8$ & $58 \pm 8$ & $<0.01$ \\
\hline $\begin{array}{l}\text { Systolic blood pressure (mm } \\
\text { Hg) }\end{array}$ & $119 \pm 10$ & $126 \pm 9$ & $<0.001$ \\
\hline $\begin{array}{l}\text { Diastolic blood pressure } \\
\text { (mm Hg) }\end{array}$ & $73 \pm 10$ & $83 \pm 8$ & $<0.001$ \\
\hline ECG parameters & & & \\
\hline Heart rate (1/min) & & & \\
\hline PQ (ms) & $67 \pm 9$ & $62 \pm 6$ & $<0.01$ \\
\hline QRS (ms) & $158 \pm 25$ & $163 \pm 25$ & 0.06 \\
\hline QTc (ms) & $98 \pm 10$ & $95 \pm 7$ & 0.1 \\
\hline
\end{tabular}

Continuous data were expressed as mean \pm SD.

$\mathrm{P}<0.001$ ) and diastolic (from $73 \pm 10$ to $83 \pm 8 \mathrm{~mm} \mathrm{Hg}$; $\mathrm{P}<0.001)$ blood pressure, $\mathrm{PQ}$ time, QTc time, and heart frequency between the two examinations, while all other parameters remained stable (table 1). No arrhythmias, abnormalities in conduction or signs of ischaemia were detected by ECG.

Laboratory values were within normal range at both visits, and the detailed data are provided in table 2. No significant changes in cardiac biomarkers (high-sensitivity troponin T and NT-proBNP) were observed.

\section{CMR results}

All CMR parameters of all subjects were within the reference range on both visits, with normal initial stress test results. On the follow-up visit, significant changes occurred in end-diastolic volume (from $155 \pm 31 \mathrm{~mL}$ to $143 \pm 28 \mathrm{~mL}, \mathrm{P}=0.01$ ), end-systolic volume (from $60 \pm 14 \mathrm{~mL}$ to $55 \pm 12 \mathrm{~mL}, \mathrm{P}=0.01$ ) and stroke volume (from $94 \pm 20 \mathrm{~mL}$ to $88 \pm 17 \mathrm{~mL}, \mathrm{P}=0.03$ ), whereas LV mass, LVEF and parameters of longitudinal function remained unchanged. All parameters are given in table 3 .

No significant change occurred in native $\mathrm{T} 1$ times (baseline: $1017 \pm 24 \mathrm{~ms}$ vs follow-up: $1015 \pm 21 \mathrm{~ms}$; $\mathrm{P}=0.6$; figure 1), postcontrast T1 times (baseline: $426 \pm 38 \mathrm{~ms}$ vs follow-up: $413 \pm 20 \mathrm{~ms} ; \mathrm{P}=0.13$; figure 2) or $\mathrm{ECV}$ (baseline: $22 \pm 2 \%$ vs follow-up: $23 \% \pm 2 \%$; $\mathrm{P}=0.3$; figure 3 ). Native T1 time and ECV were better reproducible than postcontrast $\mathrm{T} 1$, resulting in better coefficients of variation (ECV: $3.5 \%$, native $\mathrm{T} 1: 1.3 \%$, postcontrast $\mathrm{T} 1: 6.4 \%$ ). Limits of agreement were smaller in ECV and native compared with postcontrast T1 (ECV: $2 \% /-2 \%$, native T1: $39 \mathrm{~ms} /-35$ ms, postcontrast T1: $85 \mathrm{~ms} /-59 \mathrm{~ms}$ ). Bland-Altman plots are given in figure 4 .

\section{Gender-wise analysis}

Male subjects had a significantly higher body mass index $\left(26 \pm 1 \mathrm{~kg} / \mathrm{m}^{2}\right.$ vs $\left.22 \pm 1 \mathrm{~kg} / \mathrm{m}^{2}, \mathrm{P}=0.02\right)$, but did not differ
Table 2 Laboratory results

\begin{tabular}{|c|c|c|c|}
\hline & Baseline visit & Follow-up visit & $P$ value \\
\hline $\begin{array}{l}\text { High- } \\
\text { sensitivity troponin T } \\
(\mathrm{pg} / \mathrm{mL})\end{array}$ & $3.9 \pm 1.3$ & $3.9 \pm 1.1$ & 0.99 \\
\hline NT-proBNP (ng/L) & $68 \pm 44$ & $71 \pm 48$ & 0.66 \\
\hline GOT (U/L) & $21 \pm 4$ & $29 \pm 15$ & 0.03 \\
\hline GPT (U/L) & $22 \pm 9$ & $30 \pm 36$ & 0.39 \\
\hline Bilirubin (mg/dL) & $0.8 \pm 0.4$ & $0.8 \pm 0.2$ & 0.31 \\
\hline Gamma-GT (U/L) & $23 \pm 14$ & $31 \pm 39$ & 0.42 \\
\hline Creatinine (mg(dL) & $0.9 \pm 0.1$ & $0.8 \pm 0.1$ & 0.02 \\
\hline GFR (mL/min) & $96 \pm 20$ & $96 \pm 17$ & 0.90 \\
\hline Cholesterol (mg/dL) & $204 \pm 23$ & $201 \pm 33$ & 0.44 \\
\hline $\begin{array}{l}\text { HDL-cholesterol (mg/ } \\
\mathrm{dL} \text { ) }\end{array}$ & $61 \pm 15$ & $57 \pm 18$ & 0.08 \\
\hline $\begin{array}{l}\text { LDL-cholesterol (mg/ } \\
\mathrm{dL})\end{array}$ & $127 \pm 23$ & $123 \pm 28$ & 0.26 \\
\hline Haemoglobin A1c (\%) & $5.5 \pm 0.2$ & $5.3 \pm 0.2$ & 0.76 \\
\hline $\mathrm{CRP}(\mathrm{mg} / \mathrm{L})$ & $2.5 \pm 1.4$ & $2.2 \pm 0.8$ & 0.63 \\
\hline Haemoglobin $(\mathrm{g}(\mathrm{dL})$ & $14.2 \pm 1.2$ & $14.3 \pm 1.3$ & 0.45 \\
\hline Haematocrit (\%) & $42 \pm 3$ & $41 \pm 3$ & $<0.001$ \\
\hline TSH (mU/L) & $1.4 \pm 0.8$ & $1.0 \pm 0.4$ & 0.04 \\
\hline Uric acid (mg/dL) & $5 \pm 1$ & $5 \pm 1$ & 0.28 \\
\hline Sodium (mmol/L) & $141 \pm 2$ & $141 \pm 2$ & 0.76 \\
\hline Potassium (mmol/L) & $4.2 \pm 0.3$ & $4.3 \pm 0.2$ & 0.57 \\
\hline
\end{tabular}

Continuous data were expressed as mean \pm SD.

CRP, C reactive protein; GFR, glomerular filtration rate; GOT, glutamic oxaloacetic transaminase; GPT, glutamic pyruvic transaminase; Gamma-GT, Gamma-Glutamyltranferase; HDL, high-density lipoprotein; LDL, low-density lipoprotein; NTproBNP, Nterminal pro-brain natriuretic peptide; $\mathrm{TSH}$, thyroidstimulating hormone.

significantly in any other baseline parameter. No differences in CMR parameters or T1 mapping values could be observed between genders except postcontrast T1 time at baseline visit (male: $443 \pm 8 \mathrm{~ms}$, female: $388 \pm 14 \mathrm{~ms}$; $\mathrm{P}=0.002$ ). Limits of agreement were smaller in ECV and native $\mathrm{T} 1$ compared with postcontrast $\mathrm{T} 1$ in men (native T1: 24/-27 ms, postcontrast-: -95/42 ms, ECV: 2/\%-2\%) as well as in women (native T1: $60 /-51 \mathrm{~ms}$, postcontrast T1: 48/-73 ms, ECV: 2/\%-2\%). Accordingly, coefficients of variation were better for ECV and native T1 compared with postcontrast $\mathrm{T} 1$ in men (ECV: $3.0 \%$, native T1: $0.9 \%$, postcontrast T1: $6.9 \%$ ) and women (ECV: $3.9 \%$, native T1: $1.7 \%$, postcontrast $\mathrm{T} 1: 5.9 \%$ ).

\section{DISCUSSION}

In this study we could demonstrate that in a cohort of strictly selected healthy individuals, (1) native T1, postcontrast $\mathrm{T} 1$ and $\mathrm{ECV}$ did not change significantly during a long-term follow-up; (2) native T1 and ECV values showed less variation between measurements than postcontrast 

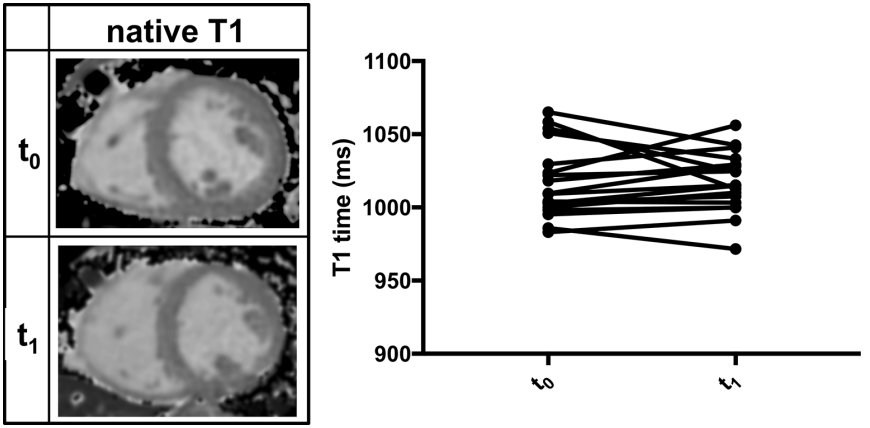

Figure 1 Representative images of native T1 maps and plots of individual T1 values at baseline $\left(\mathrm{t}_{0}\right)$ and follow-up visit $\left(\mathrm{t}_{1}\right)$.

T1; and (3) no significant differences between genders could be observed.

T1 mapping is an emerging technique and recently was used in numerous single-centre studies. Significant differences between healthy and diseased subjects could be demonstrated for a wide spectrum of cardiomyopathies, including dilated cardiomyopathy (DCM) ${ }^{45916}$ hypertrophic cardiomyopathy ${ }^{4517-20}$ and cardiac amyloidosis. ${ }^{721-23}$ Some studies also elucidated the prognostic value of T1 mapping in a cohort of patients with DCM, ${ }^{16}{ }^{24}$ lightchain amyloidosis, ${ }^{25}$ diabetes ${ }^{26}$ and patients with heart failure of different aetiologies. ${ }^{27}$ An association between ECV and short-term mortality in a heterogeneous cohort could be demonstrated by Wong et al. ${ }^{28}$ Before these research findings can be translated into clinical application, several technical aspects have to be addressed. First, various mapping sequences with specific advantages and disadvantages and-even more importantly-different normal ranges are available, and technical improvement is still ongoing. T1 results might vary between different vendors and magnetic resonance systems, ${ }^{29}$ and there is a lack of multivendor/multicentre trials. Furthermore, the field is heterogeneous regarding the use and dosage of contrast agents, cut-off values, quality management, image postprocessing and reporting. Due to the novelty of the technique and the different technical challenges, no universally valid reference values are available yet. In a consensus paper edited by an expert consensus group, it was suggested that normal values should be established at each CMR site for the local set-up. ${ }^{30}$ In a first multicentre, single-vendor approach, Piechnik et al ${ }^{13}$ provided reference values for 1.5 Tesla in a large cohort of 342 healthy volunteers. Additionally, mapping values for different cardiovascular diseases were provided. ${ }^{31}$ Dabir et $a l^{2}$ provided reference values for 1.5 and $3 \mathrm{~T}$ based on 103 healthy subjects, examined in four different centres under the same conditions using a standardised mapping sequence. Normal values of $1.5 \mathrm{~T} \mathrm{~T} 1$ mapping parameters based on smaller cohorts were also published by Messroghli et al, ${ }^{14}$ Gai et al,${ }^{33}$ Liu et al, ${ }^{34}$ von Knobelsdorf-Brenkenhoff $e t a l^{35}$ and Nacif $e t a l^{36}$ The average values in our cohort were closest to the values obtained by Nacif $e t a l$, using the same sequence with the same flip angle of $35^{\circ}$.
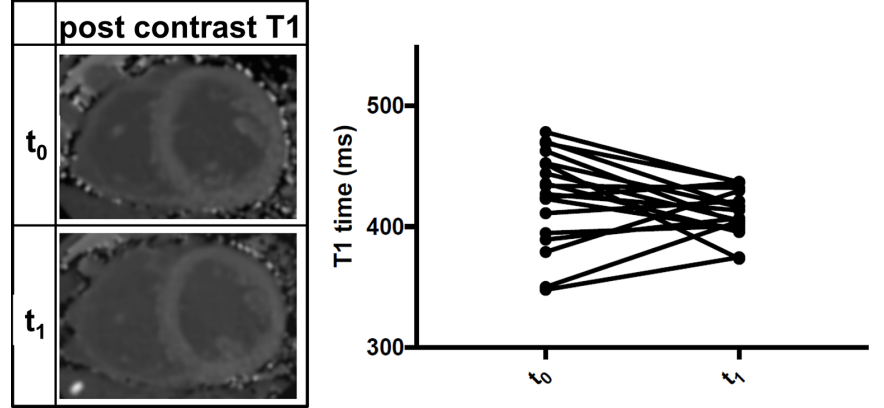

Figure 2 Representative images of postcontrast T1 maps and plots of individual postcontrast T1 values at baseline $\left(t_{0}\right)$ and follow-up visit $\left(t_{1}\right)$.

Postcontrast T1 times depend on the dosage of contrast agents, the total volume of distribution and the time between injection of contrast medium and image acquisition. This may explain why postcontrast $\mathrm{T} 1 \mathrm{had}$ the highest coefficient of variation among all parameters in our cohort and therefore appeared to be less robust than native T1 or ECV. As a derived parameter, ECV appears much less dependent on technical confounders and field strength. Additionally, ECV has a lower variability between different vendors than native and postcontrast $\mathrm{T} 1 .^{29}$ Indeed, in our study, ECV had a good

Table 3 CMR parameters

\begin{tabular}{lccl}
\hline & Baseline visit & Follow-up visit & P value \\
\hline LVEDD $(\mathrm{mm})$ & $48 \pm 4$ & $47 \pm 3$ & 0.3 \\
\hline LVESD $(\mathrm{mm})$ & $32 \pm 4$ & $32 \pm 3$ & 0.7 \\
\hline EDV $(\mathrm{mL})$ & $155 \pm 31$ & $143 \pm 28$ & 0.01 \\
\hline ESV $(\mathrm{mL})$ & $60 \pm 14$ & $55 \pm 12$ & 0.01 \\
\hline SV $(\mathrm{mL})$ & $94 \pm 20$ & $88 \pm 17$ & 0.03 \\
LVEF $(\%)$ & $61 \pm 4$ & $62 \pm 3$ & 0.4 \\
LV mass $(\mathrm{g})$ & $83 \pm 24$ & $88 \pm 26$ & 0.06 \\
MAPSE $(\mathrm{mm})$ & $12 \pm 3$ & $12 \pm 2$ & 0.9 \\
\hline TAPSE $(\mathrm{mm})$ & $20 \pm 3$ & $19 \pm 3$ & 0.5
\end{tabular}

T1 mapping

parameters

\begin{tabular}{lccc|}
$\begin{array}{l}\text { Native T1 } \\
\text { myocardium (ms) }\end{array}$ & $1017 \pm 24$ & $1015 \pm 21$ & 0.6 \\
\hline Native T1 blood (ms) & $1542 \pm 87$ & $1552 \pm 63$ & 0.6 \\
\hline $\begin{array}{l}\text { Postcontrast T1 } \\
\text { myocardium (ms) }\end{array}$ & $426 \pm 38$ & $413 \pm 20$ & 0.13 \\
$\begin{array}{l}\text { Postcontrast T1 blood } \\
\text { (ms) }\end{array}$ & $242 \pm 33$ & $233 \pm 16$ & 0.2 \\
\hline ECV (\%) & $22 \pm 2$ & $23 \pm 2$ & 0.3 \\
\hline
\end{tabular}

Continuous data were expressed as mean $\pm \mathrm{SD}$.

CMR, cardiovascular magnetic resonance;

ECV, extracellularvolume; ESV, end-systolic volume; EDV, enddiastolic volume; LV, left ventricular; LVEDD, left ventricular end-diastolic diameter; LVEF, left ventricular ejection fraction; LVESD, left ventricular end-systolic diameter; MAPSE, mitral annulus plane systolic excursion; SV, stroke volume; TAPSE, tricuspid annular plane systolic excursion. 

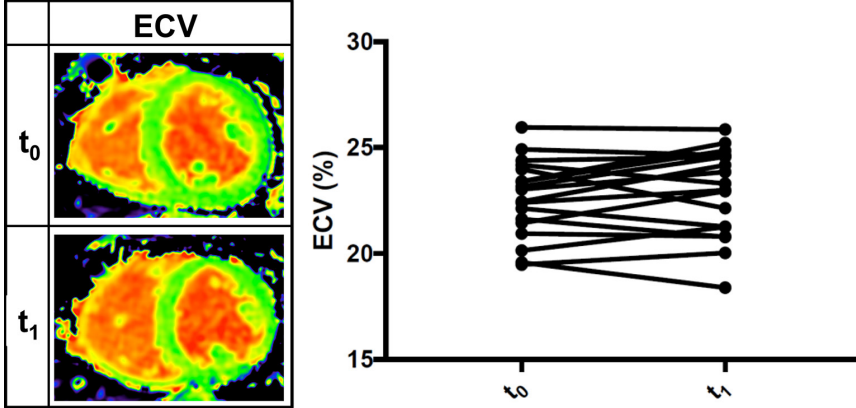

Figure 3 Representative images of extracellular volume (ECV) maps and plots of individual ECV values at baseline $\left(t_{0}\right)$ and follow-up visit $\left(t_{1}\right)$.
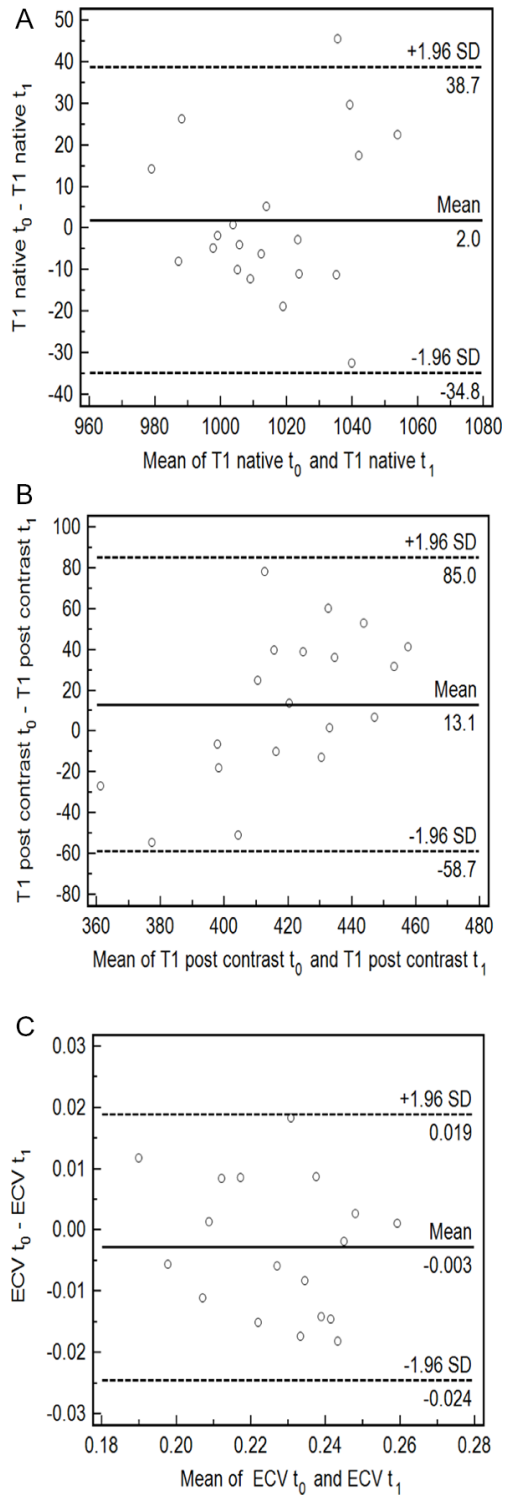

Figure 4 Bland-Altman plots of native T1 (A), postcontrast $\mathrm{T} 1 \mathrm{~B})$ and extracellular volume $(\mathrm{ECV})(\mathrm{C}) . \mathrm{T}_{0}$, baseline visit; $\mathrm{t}_{1}$, follow up visit. reproducibility, with just a slightly higher coefficient of variation than native $\mathrm{T} 1$.

In our cohort, no significant changes could be detected between baseline and follow-up scans, but several studies reported an age dependency of $\mathrm{T} 1$ mapping parameters. For instance, an increase of $\mathrm{ECV}^{2637}$ and a decrease of native $\mathrm{T} 1$ times with age ${ }^{13}$ were described. It remains unclear whether the observed changes might indicate a subclinical disease or the physiological ageing process. However, the small sample size in our study should be considered as a major limitation in this context.

The finding that native $\mathrm{T} 1$ and ECV remain constant in healthy volunteers over several years, respectively, the long-term stability of T1 mapping parameters in individuals, is an essential precondition for applications such as monitoring during therapeutic interventions or assessment of natural disease progression. Of course, our study is limited by a small case number and a lack of data for different sequences, field strengths, vendors or postprocessing procedures. Nevertheless, the presented data endorse the results of previous studies regarding stability of T1 measurements.

\section{CONCLUSION}

Myocardial T1 and ECV values are stable over a long-term follow-up period in healthy subjects and thus appear suitable for longitudinal studies. While a similar reproducibility could be demonstrated for native $\mathrm{T} 1$ and ECV, postcontrast $\mathrm{T} 1$ appeared to be less robust.

Acknowledgements We thank our medical technical assistant Daniel Helm for excellent image acquisition and his valuable and sustained support.

Contributors FadS: study conception and design, drafting of manuscript. CB: acquisition of data. MM-H: analysis and interpretation of data, drafting of manuscript. FA: analysis and interpretation of data, statistics. DM: critical revision. MO: critical revision. JR: critical revision. EG: critical revision. HAK: critical revision. MGF: critical revision. SJB: analysis and interpretation of data, drafting of manuscript.

Competing interests None declared.

Ethics approval The study was conducted in accordance with the ethical principles of the Declaration of Helsinki and was approved by the local ethics committee of the University of Heidelberg, Germany (reference number: S-038/2007, Ethikkommission Heidelberg, Alte Glockengießerei 11/1, 60015 Heidelberg).

Provenance and peer review Not commissioned; externally peer reviewed. Data sharing statement No additional data are available.

Open Access This is an Open Access article distributed in accordance with the Creative Commons Attribution Non Commercial (CC BY-NC 4.0) license, which permits others to distribute, remix, adapt, build upon this work non-commercially, and license their derivative works on different terms, provided the original work is properly cited and the use is non-commercial. See: http://creativecommons.org/ licenses/by-nc/4.0/

(C) Article author(s) (or their employer(s) unless otherwise stated in the text of the article) 2018. All rights reserved. No commercial use is permitted unless otherwise expressly granted.

\section{REFERENCES}

1. White SK, Sado DM, Flett AS, et al. Characterising the myocardial interstitial space: the clinical relevance of non-invasive imaging. Heart 2012;98:773-9. 
2. Messroghli DR, Niendorf T, Schulz-Menger J, et al. T1 mapping in patients with acute myocardial infarction. J Cardiovasc Magn Reson 2003:5:353-9.

3. Ugander M, Oki AJ, Hsu LY, et al. Extracellular volume imaging by magnetic resonance imaging provides insights into overt and subclinical myocardial pathology. Eur Heart J 2012;33:1268-78.

4. Puntmann VO, Voigt T, Chen Z, et al. Native T1 mapping in differentiation of normal myocardium from diffuse disease in hypertrophic and dilated cardiomyopathy. JACC Cardiovasc Imaging 2013;6:475-84.

5. Dass S, Suttie JJ, Piechnik SK, et al. Myocardial tissue characterization using magnetic resonance noncontrast $\mathrm{t} 1$ mapping in hypertrophic and dilated cardiomyopathy. Circ Cardiovasc Imaging 2012;5:726-33.

6. Ferreira VM, Piechnik SK, Dall'Armellina E, et al. Non-contrast T1-mapping detects acute myocardial edema with high diagnostic accuracy: a comparison to T2-weighted cardiovascular magnetic resonance. J Cardiovasc Magn Reson 2012;14:42

7. Fontana M, Banypersad SM, Treibel TA, et al. Native T1 mapping in transthyretin amyloidosis. JACC Cardiovasc Imaging 2014:7:157-65.

8. Sibley CT, Noureldin RA, Gai N, et al. T1 Mapping in cardiomyopathy at cardiac MR: comparison with endomyocardial biopsy. Radiology 2012;265:724-32.

9. aus dem Siepen F, Buss SJ, Messroghli D, et al. T1 mapping in dilated cardiomyopathy with cardiac magnetic resonance: quantification of diffuse myocardial fibrosis and comparison with endomyocardial biopsy. Eur Heart J Cardiovasc Imaging 2015;16:210-6.

10. Iles L, Pfluger H, Phrommintikul A, et al. Evaluation of diffuse myocardial fibrosis in heart failure with cardiac magnetic resonance contrast-enhanced T1 mapping. J Am Coll Cardiol 2008;52:1574-80.

11. Lee H, Park JB, Yoon YE, et al. Noncontrast Myocardial T1 Mapping by Cardiac Magnetic Resonance Predicts Outcome in Patients With Aortic Stenosis. JACC Cardiovasc Imaging 2017. doi: 10.1016/j. jcmg.2017.09.005. [Epub ahead of print 10 Nov 2017].

12. Levelt E, Piechnik SK, Liu A, et al. Adenosine stress CMR T1mapping detects early microvascular dysfunction in patients with type 2 diabetes mellitus without obstructive coronary artery disease. J Cardiovasc Magn Reson 2017;19:81.

13. Piechnik SK, Ferreira VM, Lewandowski AJ, et al. Normal variation of magnetic resonance $\mathrm{T} 1$ relaxation times in the human population at 1.5 T using ShMOLLI. J Cardiovasc Magn Reson 2013;15:13.

14. Messroghli DR, Greiser A, Fröhlich M, et al. Optimization and validation of a fully-integrated pulse sequence for modified looklocker inversion-recovery (MOLLI) T1 mapping of the heart. J Magn Reson Imaging 2007;26:1081-6.

15. Flett AS, Hayward MP, Ashworth MT, et al. Equilibrium contrast cardiovascular magnetic resonance for the measurement of diffuse myocardial fibrosis: preliminary validation in humans. Circulation 2010;122:138-44.

16. Barison A, Grigoratos C, Todiere G, et al. Myocardial interstitial remodelling in non-ischaemic dilated cardiomyopathy: insights from cardiovascular magnetic resonance. Heart Fail Rev 2015;20:731-49.

17. Kellman $\mathrm{P}$, Wilson JR, Xue $\mathrm{H}$, et al. Extracellular volume fraction mapping in the myocardium, part 2: initial clinical experience. $J$ Cardiovasc Magn Reson 2012;14:64.

18. Sado DM, Flett AS, Banypersad SM, et al. Cardiovascular magnetic resonance measurement of myocardial extracellular volume in health and disease. Heart 2012;98:1436-41.

19. Kramer CM, Chandrashekhar Y, Narula J. T1 mapping by CMR in cardiomyopathy: a noninvasive myocardial biopsy? JACC Cardiovasc Imaging 2013;6:532-4.

20. Mordi I, Carrick D, Bezerra H, et al. T1 and T2 mapping for early diagnosis of dilated non-ischaemic cardiomyopathy in middle-aged patients and differentiation from normal physiological adaptation. Eur Heart J Cardiovasc Imaging 2016;17:797-803.
21. Mongeon FP, Jerosch-Herold M, Coelho-Filho OR, et al. Quantification of extracellular matrix expansion by CMR in infiltrative heart disease. JACC Cardiovasc Imaging 2012;5:897-907.

22. Fontana M, Pica S, Reant P, et al. Prognostic Value of Late Gadolinium Enhancement Cardiovascular Magnetic Resonance in Cardiac Amyloidosis. Circulation 2015;132:1570-9.

23. Banypersad SM, Sado DM, Flett AS, et al. Quantification of myocardial extracellular volume fraction in systemic $A L$ amyloidosis: an equilibrium contrast cardiovascular magnetic resonance study. Circ Cardiovasc Imaging 2013;6:34-9.

24. Puntmann VO, Carr-White G, Jabbour A, et al. T1-Mapping and Outcome in Nonischemic Cardiomyopathy: All-Cause Mortality and Heart Failure. JACC Cardiovasc Imaging 2016;9:40-50.

25. Banypersad SM, Fontana M, Maestrini V, et al. T1 mapping and survival in systemic light-chain amyloidosis. Eur Heart $J$ 2015;36:244-51.

26. Wong TC, Piehler KM, Kang IA, et al. Myocardial extracellular volume fraction quantified by cardiovascular magnetic resonance is increased in diabetes and associated with mortality and incident heart failure admission. Eur Heart J 2014;35:657-64.

27. Schelbert EB, Piehler KM, Zareba KM, et al. Myocardial Fibrosis quantified by extracellular volume is associated with subsequent hospitalization for heart failure, death, or both across the spectrum of ejection fraction and heart failure stage. J Am Heart Assoc 2015;4:e002613.

28. Wong TC, Piehler K, Meier CG, et al. Association between extracellular matrix expansion quantified by cardiovascular magnetic resonance and short-term mortality. Circulation 2012;126:1206-16.

29. Raman FS, Kawel-Boehm N, Gai N, et al. Modified look-locker inversion recovery T1 mapping indices: assessment of accuracy and reproducibility between magnetic resonance scanners. J Cardiovasc Magn Reson 2013;15:64.

30. Moon JC, Messroghli DR, Kellman P, et al. Society for Cardiovascular Magnetic Resonance I and Cardiovascular Magnetic Resonance Working Group of the European Society of C. Myocardial T1 mapping and extracellular volume quantification: a Society for Cardiovascular Magnetic Resonance (SCMR) and CMR Working Group of the European Society of Cardiology consensus statement. $J$ Cardiovasc Magn Reson 2013;15:92.

31. Liu JM, Liu A, Leal J, et al. Measurement of myocardial native T1 in cardiovascular diseases and norm in 1291 subjects. J Cardiovasc Magn Reson 2017;19:74.

32. Dabir D, Child N, Kalra A, et al. Reference values for healthy human myocardium using a T1 mapping methodology: results from the International T1 Multicenter cardiovascular magnetic resonance study. J Cardiovasc Magn Reson 2014;16:69.

33. Gai ND, Stehning C, Nacif M, et al. Modified Look-Locker T1 evaluation using Bloch simulations: human and phantom validation. Magn Reson Med 2013;69:329-36.

34. Liu S, Han J, Nacif MS, et al. Diffuse myocardial fibrosis evaluation using cardiac magnetic resonance $\mathrm{T} 1$ mapping: sample size considerations for clinical trials. J Cardiovasc Magn Reson 2012;14:90.

35. von Knobelsdorff-Brenkenhoff F, Prothmann M, Dieringer MA, et al. Myocardial T1 and T2 mapping at 3 T: reference values, influencing factors and implications. J Cardiovasc Magn Reson 2013;15:53

36. Nacif MS, Turkbey EB, Gai N, et al. Myocardial T1 mapping with MRI: comparison of look-locker and MOLLI sequences. J Magn Reson Imaging 2011;34:1367-73.

37. Liu CY, Liu YC, Wu C, et al. Evaluation of age-related interstitial myocardial fibrosis with cardiac magnetic resonance contrast-enhanced T1 mapping: MESA (Multi-Ethnic Study of Atherosclerosis). J Am Coll Cardiol 2013;62:1280-7. 